\title{
EXACT FORMULAS FOR COEFFICIENTS OF JACOBI FORMS
}

\author{
KATHRIN BRINGMANN*,‡ and OLAV K. RICHTER ${ }^{\dagger, \S}$ \\ *Mathematisches Institut, Universität Köln \\ Weyertal 86-90, D-50931 Köln, Germany \\ †Department of Mathematics, University of North Texas \\ Denton, TX 76203, USA \\ $\ddagger_{k b r i n g m a @ m a t h . u n i-k o e l n . d e}$ \\ §richter@unt.edu
}

Received 2 June 2010

Accepted 21 July 2010

\begin{abstract}
In previous work, we introduced harmonic Maass-Jacobi forms. The space of such forms includes the classical Jacobi forms and certain Maass-Jacobi-Poincaré series, as well as Zwegers' real-analytic Jacobi forms, which play an important role in the study of mock theta functions and related objects. Harmonic Maass-Jacobi forms decompose naturally into holomorphic and non-holomorphic parts. In this paper, we give exact formulas for the Fourier coefficients of the holomorphic parts of harmonic Maass-Jacobi forms and, in particular, we obtain explicit formulas for the Fourier coefficients of weak Jacobi forms.
\end{abstract}

Keywords: Jacobi forms; harmonic Maass-Jacobi forms; mock theta functions.

Mathematics Subject Classification 2010: 11F50, 11F30, 11F37

\section{Introduction and Statement of Results}

In 1985, Eichler and Zagier [5] systematically developed a theory of Jacobi forms. That theory has since grown enormously, establishing deep connections to many other areas of mathematics and physics, such as the theory of Heegner points (see [6]), the theory of elliptic genera (see [10]), string theory (see, for example, [4]), and more recently, mock theta functions (see [11]). In [2], we initiated a theory of harmonic Maass-Jacobi forms, which includes the holomorphic Jacobi forms of [5], the real-analytic Jacobi forms in [11], and certain Maass-Jacobi-Poincaré series as explicit examples. Of particular interest is $\widehat{\mathbb{J}}_{k, m}^{\text {cusp }}$, a distinguished subspace of harmonic Maass-Jacobi forms, which we will briefly review in Sec. 2. If $\phi \in \widehat{\mathbb{J}}_{k, m}^{\text {cusp }}$, then $\phi=\phi^{+}+\phi^{-}$, where

$$
\phi^{+}(\tau, z)=\sum_{\substack{n, r \in \mathbb{Z} \\ D \ll \infty}} c^{+}(n, r) q^{n} \zeta^{r}
$$


is the holomorphic part of $\phi$, while the non-holomorphic part of $\phi$ is given by

$$
\phi^{-}(\tau, z)=\sum_{\substack{n, r \in \mathbb{Z} \\ D>0}} c^{-}(n, r) \Gamma\left(\frac{3}{2}-k, \frac{\pi D y}{m}\right) q^{n} \zeta^{r} .
$$

Here and throughout the paper, $\tau=x+i y \in \mathbb{H}$ (the usual complex upper halfplane), $z=u+i v \in \mathbb{C}, q:=e^{2 \pi i \tau}, \zeta:=e^{2 \pi i z}, D:=r^{2}-4 n m$, and $\Gamma(s, x):=$ $\int_{x}^{\infty} e^{-t} t^{s-1} d t$ is the incomplete Gamma-function. In particular, if $\phi \in \widehat{\mathbb{J}}_{k, m}^{\text {cusp }}$ is holomorphic, then $\phi=\phi^{+}$is a weak Jacobi form. If, in addition, the Fourier series in (1) is only over $D \leq 0$, then $\phi$ is a Jacobi form as in [5] and if the Fourier series in (1) is only over $D<0$, then $\phi$ is a Jacobi cusp form. Note that our notion of weak Jacobi form is slightly more general then the one in [5], where weak Jacobi forms have an expansion as in (1) with the additional condition that $n \geq 0$. If $\phi^{+}$ is as in (1), then we call

$$
P_{\phi^{+}}(\tau, z):=\sum_{\substack{n, r \in \mathbb{Z} \\ D>0}} c^{+}(n, r) q^{n} \zeta^{r}
$$

the principal part of $\phi^{+}$.

In this paper, we extend ideas of [1] to obtain exact formulas for the coefficients of $\phi^{+}$in (1). It turns out that the principal part $P_{\phi^{+}}$in (3) dictates the coefficients of $\phi^{+}$for $D<0$, as explained in the following theorem, which is the main result of this paper.

Theorem 1. Let $\phi \in \widehat{\mathbb{J}}_{k, m}^{\text {cusp }}$ with holomorphic part $\phi^{+}$as in (1). If $k<0$ is even and $D^{\prime}:=r^{\prime 2}-4 m n^{\prime}<0$, then

$$
c^{+}\left(n^{\prime}, r^{\prime}\right)=\frac{1}{2 \Gamma\left(\frac{5}{2}-k\right)} \sum_{r(\underset{D>0}{\bmod 2 m)}} c^{+}(n, r) c_{n, r}^{(k)}\left(n^{\prime}, r^{\prime}\right)
$$

where $\Gamma(\cdot)$ is the Gamma-function and

$$
c_{n, r}^{(k)}\left(n^{\prime}, r^{\prime}\right):=b_{n, r}^{(k)}\left(n^{\prime}, r^{\prime}\right)+(-1)^{k} b_{n, r}^{(k)}\left(n^{\prime},-r^{\prime}\right)
$$

with

$$
\begin{aligned}
b_{n, r}^{(k)}\left(n^{\prime}, r^{\prime}\right):= & \sqrt{2} \pi i^{-k} m^{-\frac{1}{2}}\left(\frac{\left|D^{\prime}\right|}{D}\right)^{\frac{k}{2}-\frac{3}{4}} \\
& \times \sum_{c>0} c^{-\frac{3}{2}} K_{c}\left(n, r, n^{\prime}, r^{\prime}\right) I_{k-\frac{3}{2}}\left(\frac{\pi \sqrt{\left|D^{\prime}\right| D}}{m c}\right) .
\end{aligned}
$$

Here $I_{s}(x)$ is the usual I-Bessel function of order $s$ and $K_{c}\left(n, r, n^{\prime}, r^{\prime}\right)$ is the Kloosterman sum

$$
K_{c}\left(n, r, n^{\prime}, r^{\prime}\right):=e_{2 m c}\left(-r r^{\prime}\right) \sum_{\substack{d(\bmod c)^{*} \\ \lambda(\bmod c)}} e_{c}\left(\bar{d} m \lambda^{2}+n^{\prime} d-r^{\prime} \lambda+\bar{d} n+\bar{d} r \lambda\right),
$$


where $e_{c}(x):=e^{\frac{2 \pi i x}{c}}$ and the sum over $d$ runs through the primitive residue classes modulo $c$ and $\bar{d}$ is the inverse of $d$ modulo $c$.

In Sec. 2, we follow [3] to define a pairing of harmonic Maass--Jacobi forms and skew-holomorphic Jacobi forms. We find that this pairing is determined by the principal part of the holomorphic part of a harmonic Maass-Jacobi form. In Sec. 3, we recall the Maass-Jacobi-Poincaré series from [2]. Given a harmonic MaassJacobi form, linear combinations of such Poincaré series allow one to construct a new harmonic Maass-Jacobi form whose holomorphic part has the same principal part as the given one. This is the key idea in our proof of Theorem 1. Finally, we give an explicit application of our results. The ring of Jacobi forms of even weights is generated (over the ring of modular forms) by certain weak Jacobi forms of index 1 and weights -2 and 0 , which we denote by $\phi_{-2,1}$ and $\phi_{0,1}$, respectively (see [5, $\S \S 8$ and 9]). We show that $\phi_{-2,1}$ can be expressed as Jacobi-Poincaré series. It is likely that $\phi_{0,1}$ can also be realized as a Jacobi-Poincaré series, but this requires the existence of a Maass-Jacobi-Poincaré series of weight 0 , which we have not constructed yet.

\section{The Pairing}

In this section, we introduce a pairing between skew-holomorphic Jacobi forms and harmonic Maass-Jacobi forms, which is vital to our proof of Theorem 1 in Sec. 3. Let $J_{k, m}^{s k}$ denote the space of skew-holomorphic Jacobi forms of weight $k$ and index $m$ and let $J_{k, m}^{s k \text {,cusp }}$ be the subspace of cusp forms (for details, see [8,9]). If $\phi, \psi \in J_{k, m}^{s k, \text { cusp }}$, then the Petersson scalar product of $\phi$ and $\psi$ is defined by

$$
\langle\phi, \psi\rangle:=\int_{\Gamma^{J} \backslash \mathbb{H} \times \mathbb{C}} \phi(\tau, z) \overline{\psi(\tau, z)} e^{-\frac{4 \pi m v^{2}}{y}} y^{k} d V,
$$

where $\Gamma^{J}:=\mathrm{SL}_{2}(\mathbb{Z}) \ltimes \mathbb{Z}^{2}$ is the Jacobi group and $d V:=\frac{d u d v d x d y}{y^{3}}$ is the $\Gamma^{J}$-invariant volume element on $\mathbb{H} \times \mathbb{C}$ (for details, see [7, 9]). Note that skew-holomorphic Jacobi forms have a theta decomposition, i.e. if

$$
\phi(\tau, z)=\sum_{\substack{n, r \in \mathbb{Z} \\ D \geq 0}} c(n, r) e^{-\frac{\pi D y}{m}} q^{n} \zeta^{r} \in J_{k, m}^{s k},
$$

then

$$
\phi(\tau, z)=\sum_{\mu(\bmod 2 m)} h_{\mu}(\tau) \theta_{m, \mu}(\tau, z),
$$

where

$$
h_{\mu}(\tau):=\sum_{N \geq 0} c_{\mu}(N) \bar{q}^{\frac{N}{4 m}}
$$


with

$$
c_{\mu}(N):= \begin{cases}c\left(\frac{r^{2}-N}{4 m}, r\right) & \text { for } r \in \mathbb{Z}, r \equiv \mu(\bmod 2 m), \text { and } N \equiv \mu^{2}(\bmod 4 m), \\ 0 & \text { if } N \not \equiv \mu^{2}(\bmod 4 m)\end{cases}
$$

and

$$
\theta_{m, \mu}(\tau, z):=\sum_{\substack{r \in \mathbb{Z} \\ r \equiv \mu(\bmod 2 m)}} q^{\frac{r^{2}}{4 m}} \zeta^{r}
$$

If

$$
\phi(\tau, z)=\sum_{\mu(\bmod 2 m)} h_{\mu}(\tau) \theta_{m, \mu}(\tau, z) \in J_{k, m}^{s k, \text { cusp }}
$$

and

$$
\psi(\tau, z)=\sum_{\mu(\bmod 2 m)} g_{\mu}(\tau) \theta_{m, \mu}(\tau, z) \in J_{k, m}^{s k, \text { cusp }},
$$

then analogous to Theorem 5.3 of [5], one finds that

$$
\langle\phi, \psi\rangle=\int_{\mathcal{F}} \sum_{\mu(\bmod 2 m)} h_{\mu}(\tau) \overline{g_{\mu}(\tau)} y^{k-\frac{5}{2}} d x d y,
$$

where $\mathcal{F}$ is the standard fundamental domain for the action of $\mathrm{SL}_{2}(\mathbb{Z})$ on $\mathbb{H}$.

We wish to review the definition of harmonic Maass-Jacobi forms of [2]. Therefore, we need to recall the slash action for Jacobi forms and a certain differential operator which is invariant under that action. If $\phi: \mathbb{H} \times \mathbb{C} \rightarrow \mathbb{C}$, then

$$
\begin{aligned}
& \left(\left.\phi\right|_{k, m} A\right)(\tau, z) \\
& \quad:=\phi\left(\frac{a \tau+b}{c \tau+d}, \frac{z+\lambda \tau+\mu}{c \tau+d}\right)(c \tau+d)^{-k} e^{2 \pi i m\left(\frac{-c(z+\lambda \tau+\mu)^{2}}{c \tau+d}+\lambda^{2} \tau+2 \lambda z\right)}
\end{aligned}
$$

for all $A=\left[\left(\begin{array}{ll}a & b \\ c & d\end{array}\right),(\lambda, \mu)\right] \in \Gamma^{J}$. Furthermore, if $\partial_{w}:=\frac{\partial}{\partial w}$ for a variable $w$, then

$$
\begin{aligned}
& C^{k, m}:=-2(\tau-\bar{\tau})^{2} \partial_{\tau \bar{\tau}}-(2 k-1)(\tau-\bar{\tau}) \partial_{\bar{\tau}}+\frac{(\tau-\bar{\tau})^{2}}{4 \pi i m} \partial_{\bar{\tau} z z}+\frac{k(\tau-\bar{\tau})}{4 \pi i m} \partial_{z \bar{z}} \\
& +\frac{(\tau-\bar{\tau})(z-\bar{z})}{4 \pi i m} \partial_{z z \bar{z}}-2(\tau-\bar{\tau})(z-\bar{z}) \partial_{\tau \bar{z}}+(1-k)(z-\bar{z}) \partial_{\bar{z}} \\
& +\frac{(\tau-\bar{\tau})^{2}}{4 \pi i m} \partial_{\tau \overline{z z}}+\left(\frac{(z-\bar{z})^{2}}{2}+\frac{k(\tau-\bar{\tau})}{4 \pi i m}\right) \partial_{\overline{z z}}+\frac{(\tau-\bar{\tau})(z-\bar{z})}{4 \pi i m} \partial_{z \overline{z z}}
\end{aligned}
$$

which (up to the constant $\frac{5}{8}+\frac{3 k-k^{2}}{2}$ ) is the Casimir operator with respect to the action in (9). There is a misprint in [2]: the term $k(z-\bar{z}) \partial_{\bar{z}}$ should be $(1-k)(z-\bar{z}) \partial_{\bar{z}}$.

Definition 1. A function $\phi: \mathbb{H} \times \mathbb{C} \rightarrow \mathbb{C}$ is a harmonic Maass-Jacobi form of weight $k$ and index $m$ if $\phi$ is real-analytic in $\tau \in \mathbb{H}$ and $z \in \mathbb{C}$ and satisfies the 
following conditions:

(1) For all $A \in \Gamma^{J},\left(\left.\phi\right|_{k, m} A\right)=\phi$.

(2) We have that $C^{k, m}(\phi)=0$.

(3) We have that $\phi(\tau, z)=O\left(e^{a y} e^{\frac{2 \pi m v^{2}}{y}}\right)$ as $y \rightarrow \infty$ for some $a>0$.

Of particular interest are harmonic Maass-Jacobi forms, which are holomorphic in $z$; the space of such forms is denoted by $\widehat{\mathbb{J}}_{k, m}$.

The differential operator

$$
\xi_{k, m}:=y^{k-\frac{3}{2}}\left(-2 i y \partial_{\bar{\tau}}-2 i v \partial_{\bar{z}}+\frac{y}{4 \pi m} \partial_{\overline{z z}}\right)
$$

plays an important role in this context. It maps harmonic Maass--Jacobi forms that are holomorphic in the Jacobi variable $z$ to skew-holomorphic Jacobi forms. Let $\widehat{\mathbb{J}}_{k, m}^{\text {cusp }} \subset \widehat{\mathbb{J}}_{k, m}$ denote the pre-image of the space of skew-holomorphic Jacobi cusp forms under $\xi_{k, m}$. In [2], we construct explicit examples of $\widehat{\mathbb{J}}_{k, m}^{\text {cusp }}$ via Maass-JacobiPoincaré series (see (12) for the definition) and we show that $\xi_{k, m}$ maps these Maass-Jacobi-Poincaré series to skew-holomorphic Jacobi-Poincaré series, which implies the surjectivity of the map $\xi_{k, m}$ in the following proposition.

Proposition 1 ([2]). The map

$$
\xi_{k, m}: \widehat{\mathbb{J}}_{k, m}^{\text {cusp }} \rightarrow J_{3-k, m}^{s k, \text { cusp }}
$$

is surjective.

If $\phi \in \widehat{J}_{k, m}^{\text {cusp }}$ and $\psi \in J_{3-k, m}^{s k, \text { cusp }}$, then we define the (non-degenerate) pairing

$$
\{\phi, \psi\}:=\left\langle\xi_{k, m}(\phi), \psi\right\rangle \text {. }
$$

The next result extends [3, Proposition 3.5] to Jacobi forms and shows that the pairing in (10) is determined by the principal part $P_{\phi^{+}}$of $\phi^{+}$.

Proposition 2. Let $\phi=\phi^{+}+\phi^{-} \in \widehat{\mathbb{J}}_{k, m}^{\text {cusp }}$ with $\phi^{+}$and $\phi^{-}$as in (1) and (2) and let

$$
\psi(\tau, z)=\sum_{\substack{n, r \in \mathbb{Z} \\ D>0}} d(n, r) e^{-\frac{\pi D y}{m}} q^{n} \zeta^{r} \in J_{3-k, m}^{s k, \text { cusp }} .
$$

If $k$ is even, then

$$
\{\phi, \psi\}=\sum_{\substack{r(\bmod 2 m) \\ D>0}} c^{+}(n, r) \overline{d(n, r)} .
$$

Proof. Let

$$
\psi(\tau, z)=\sum_{\mu(\bmod 2 m)} g_{\mu}(\tau) \theta_{m, \mu}(\tau, z)
$$


with

$$
g_{\mu}(\tau)=\sum_{N>0} d_{\mu}(N) \bar{q}^{\frac{N}{4 m}}
$$

be the theta decomposition of $\psi$. It is easy to see that elements in $\widehat{\mathbb{J}}_{k, m}^{\text {cusp }}$ and hence also their holomorphic and non-holomorphic parts have theta decompositions. More precisely, we have

$$
\phi(\tau, z)=\sum_{\mu(\bmod 2 m)} h_{\mu}(\tau) \theta_{m, \mu}(\tau, z)
$$

where $h_{\mu}=h_{\mu}^{+}+h_{\mu}^{-}$,

$$
\begin{aligned}
& h_{\mu}^{+}(\tau)=\sum_{N \ll \infty} c_{\mu}^{+}(N) q^{-\frac{N}{4 m}}, \\
& h_{\mu}^{-}(\tau)=\sum_{N>0} c_{\mu}^{-}(N) \Gamma\left(\frac{3}{2}-k, \frac{\pi N y}{m}\right) q^{-\frac{N}{4 m}},
\end{aligned}
$$

and $c_{\mu}^{ \pm}(N)$ is as in (6).

We now follow the proof of [3, Proposition 3.5]. First, if $k$ is even, then one verifies that

$$
\sum_{\mu(\bmod 2 m)} h_{\mu} \overline{g_{\mu}} d \tau \quad \text { is an } \mathrm{SL}_{2}(\mathbb{Z}) \text {-invariant } 1 \text {-form on } \mathbb{H} \text {. }
$$

Set $L_{k}:=-2 i y^{2} \partial_{\bar{\tau}}$ and $\xi_{k}:=y^{k-2} \overline{L_{k}}$. From (8) we have

$$
\{\phi, \psi\}=\lim _{t \rightarrow \infty} \frac{1}{4 m} \sum_{j=0}^{4 m-1} \int_{\mathcal{F}_{t, j}} \sum_{\mu(\bmod 2 m)} \overline{\xi_{k-\frac{1}{2}}\left(h_{\mu}\right)(\tau) g_{\mu}(\tau)} y^{\frac{1}{2}-k} d x d y
$$

where each

$$
\begin{array}{r}
\mathcal{F}_{t, j}:=\left\{\tau \in \mathbb{H}|| \tau-j \mid \geq 1,0 \leq x-j \leq \frac{1}{2}, y \leq t,\right. \text { or } \\
\left.|\tau-j-1| \geq 1, \frac{1}{2} \leq x-j \leq 1, y \leq t\right\}
\end{array}
$$

is a translation by $j$ of the truncated fundamental domain $\mathcal{F}_{t}:=\{\tau \in \mathcal{F} \mid y \leq t\}$, and where, in addition, the "left half" is "cut and pasted" to the right. Let $d \omega:=\frac{d x d y}{y^{2}}$ denote the usual invariant volume form on $\mathbb{H}$. With the help of Stokes' theorem, we find that

$$
\begin{gathered}
\frac{1}{4 m} \sum_{j=0}^{4 m-1} \int_{\mathcal{F}_{t, j}} \sum_{\mu(\bmod 2 m)} \overline{\xi_{k-\frac{1}{2}}\left(h_{\mu}\right)(\tau) g_{\mu}(\tau)} y^{\frac{1}{2}-k} d x d y \\
=\frac{1}{4 m} \sum_{j=0}^{4 m-1} \int_{\mathcal{F}_{t, j}} \sum_{\mu(\bmod 2 m)} L_{k-\frac{1}{2}}\left(h_{\mu}\right) \overline{g_{\mu}} d \omega
\end{gathered}
$$




$$
\begin{aligned}
\text { Stokes } & \frac{-1}{4 m} \sum_{j=0}^{4 m-1} \int_{\partial \mathcal{F}_{t, j}} \sum_{\mu(\bmod 2 m)} h_{\mu} \overline{g_{\mu}} d \tau \\
& =\frac{1}{(11)} \int_{0}^{4 m} \sum_{\mu(\bmod 2 m)} h_{\mu}(x+i t) \overline{g_{\mu}(x+i t)} d x \\
& =\sum_{\substack{4 m \\
N>0}} c_{\mu}^{+}(N) \overline{d_{\mu}(N)}+\sum_{\substack{\mu(\bmod 2 m) \\
N>0}} c_{\mu}^{-}(N) \overline{d_{\mu}(N)} \Gamma\left(\frac{3}{2}-k, \frac{\pi N t}{m}\right),
\end{aligned}
$$

where the last equation follows from inserting the Fourier expansions of $h_{\mu}$ and $g_{\mu}$. Finally,

$$
\Gamma\left(\frac{3}{2}-k, \frac{\pi N t}{m}\right)=O\left(\left(\frac{\pi N t}{m}\right)^{\frac{1}{2}-k} e^{-\frac{\pi N t}{m}}\right) \quad \text { as } t \rightarrow \infty,
$$

which shows that

$$
\{\phi, \psi\}=\sum_{\substack{\mu(\bmod 2 m) \\ N>0}} c_{\mu}^{+}(N) \overline{d_{\mu}(N)}=\sum_{\substack{r(\bmod 2 m) \\ D>0}} c^{+}(n, r) \overline{d(n, r)} .
$$

\section{Maass-Jacobi-Poincaré Series and the Proof of Theorem 1}

In [2], we investigated Maass-Jacobi-Poincaré series, which we will now recall after introducing necessary notation. Let $M_{\nu, \mu}$ be the usual $M$-Whittaker function. Let $D=r^{2}-4 n m \neq 0$, and for $s \in \mathbb{C}, \kappa \in \frac{1}{2} \mathbb{Z}$, and $t \in \mathbb{R} \backslash\{0\}$, define

$$
\mathcal{M}_{s, \kappa}(t):=|t|^{-\frac{\kappa}{2}} M_{\operatorname{sgn}(t) \frac{\kappa}{2}, s-\frac{1}{2}}(|t|)
$$

and

$$
\phi_{k, m, s}^{(n, r)}(\tau, z):=\mathcal{M}_{s, k-\frac{1}{2}}\left(-\frac{\pi D y}{m}\right) e^{2 \pi i r z-\frac{\pi r^{2} y}{2 m}+2 \pi i n x} .
$$

Set $\Gamma_{\infty}^{J}:=\left\{\left[\left(\begin{array}{ll}1 & \eta \\ 0 & 1\end{array}\right),(0, n)\right] \mid \eta, n \in \mathbb{Z}\right\}$. Then the Poincaré series

$$
P_{k, m, s}^{(n, r)}(\tau, z):=\sum_{A \in \Gamma_{\infty}^{J} \backslash \Gamma^{J}}\left(\left.\phi_{k, m, s}^{(n, r)}\right|_{k, m} A\right)(\tau, z)
$$

converges absolutely and uniformly for $\operatorname{Re}(s)>\frac{5}{4}$ and $P_{k, m, s}^{(n, r)} \in \widehat{\mathbb{J}}_{k, m}$ if $s=\frac{k}{2}-\frac{1}{4}, k>$ 3 or if $s=\frac{5}{4}-\frac{k}{2}, k<0$. In [2], we determined the Fourier expansion of $P_{k, m, s}^{(n, r)}$, which features a certain theta series $\vartheta_{k, m}^{(r)}$. It is not difficult to see that

$$
\vartheta_{k, m}^{(r)}(\tau, z)=q^{-\frac{r^{2}}{4 m}}\left(\theta_{m, r}(\tau, z)+(-1)^{k} \theta_{m,-r}(\tau, z)\right) .
$$

For brevity, we will only recall the part of the Fourier expansion, which is needed for the purpose of this paper. Let $P_{k, m, s}^{(n, r)^{+}}$denote the holomorphic part of $P_{k, m, s}^{(n, r)}$. 
If $D>0$ and $s=\frac{5}{4}-\frac{k}{2}, k<0$, then [2, Corollary 1] and (13) imply that

$$
\begin{aligned}
P_{k, m, s}^{(n, r)}+ & \Gamma\left(\frac{5}{2}-k\right) q^{-\frac{D}{4 m}}\left(\theta_{m, r}(\tau, z)+(-1)^{k} \theta_{m,-r}(\tau, z)\right) \\
& +\sum_{\substack{n^{\prime}, r^{\prime} \in \mathbb{Z} \\
D^{\prime} \leq 0}} c_{n, r}^{(k)}\left(n^{\prime}, r^{\prime}\right) q^{n^{\prime}} \zeta^{r^{\prime}},
\end{aligned}
$$

where (as before) $D^{\prime}=r^{\prime 2}-4 n^{\prime} m$. If $D^{\prime}<0$, then $c_{n, r}^{(k)}\left(n^{\prime}, r^{\prime}\right)$ is as in (4).

Proof of Theorem 1. Let $\phi \in \widehat{\mathbb{J}}_{k, m}^{\text {cusp }}(k<0)$ with holomorphic part $\phi^{+}$as in (1) and principal part

$$
P_{\phi^{+}}(\tau, z)=\sum_{\substack{\mu(\bmod 2 m) \\ N>0}} c_{\mu}^{+}(N) q^{-\frac{N}{4 m}} \theta_{m, \mu}(\tau, z)
$$

with $c_{\mu}^{+}(N)$ as in (6). Equation (14), together with the identity $c_{-\mu}^{+}(N)=$ $(-1)^{k} c_{\mu}^{+}(N)$, shows that the holomorphic part of

$$
\begin{aligned}
& \varphi:=\frac{1}{2 \Gamma\left(\frac{5}{2}-k\right)} \sum_{r(\underset{D>0}{\bmod 2 m)}} c^{+}(n, r) P_{k, m, \frac{5}{4}-\frac{k}{2}}^{(n, r)} \\
& =\frac{1}{2 \Gamma\left(\frac{5}{2}-k\right)} \sum_{\substack{\bmod 2 m) \\
N>0}} c_{\mu}^{+}(N) P_{k, m, \frac{5}{4}-\frac{k}{2}}^{\left(\frac{\mu^{2}-N}{4 m}, \mu\right)}
\end{aligned}
$$

has the same principal part as $\phi^{+}$. Consider $\Phi:=\phi-\varphi \in \widehat{\mathbb{J}}_{k, m}^{\text {cusp }}$. Suppose that $\Phi$ has a non-trivial non-holomorphic part. Then $\xi_{k, m}(\Phi) \neq 0$ and hence $\left\{\Phi, \xi_{k, m}(\Phi)\right\}=$ $\left\langle\xi_{k, m}(\Phi), \xi_{k, m}(\Phi)\right\rangle \neq 0$. On the other hand, Proposition 2 expresses $\left\{\Phi, \xi_{k, m}(\Phi)\right\}$ in terms of the principal part of $\Phi$, which is zero by construction. This gives a contradiction and thus $\Phi$ is necessarily holomorphic, i.e. $\Phi$ is a weak Jacobi form. Again, the principal part of $\Phi$ is zero and hence $\Phi$ is a holomorphic Jacobi form (as in [5]) of weight $k<0$. We conclude that $\Phi=0$ and Theorem 1 follows.

We end with an explicit example.

Example 1. Let $\phi_{-2,1}(\tau, z)=\zeta-2+\zeta^{-1}+\cdots$ be the weak Jacobi form of weight -2 and index 1 alluded to in Sec. 1 . Note that

$$
\sum_{\substack{n, r \in \mathbb{Z} \\ D=1}} q^{n} \zeta^{r}=\sum_{r \equiv 1(\bmod 2)} q^{\frac{r^{2}-1}{4}} \zeta^{r}=q^{-\frac{1}{4}} \theta_{1,1}(\tau, z)
$$

appears as the principal part of $\phi_{-2,1}(\tau, z)$ and also of $\frac{1}{2 \Gamma\left(\frac{9}{2}\right)} P_{-2,1, \frac{9}{4}}^{(0,1)}(\tau, z)$. Hence we find that

$$
\phi_{-2,1}=\frac{1}{2 \Gamma\left(\frac{9}{2}\right)} P_{-2,1, \frac{9}{4}}^{(0,1)}
$$




\section{Acknowledgments}

The first author was partially supported by NSF grant DMS-0757907 and by the Alfried Krupp prize. The paper was written while the second author was in residence at RWTH Aachen University and at the Max Planck Institute for Mathematics in Bonn. He is grateful for the hospitality of each institution and he thanks Aloys Krieg in particular for providing a stimulating research environment at RWTH Aachen University.

\section{References}

[1] K. Bringmann and K. Ono, Coefficients of harmonic weak Maass forms, to appear in the Proc. of the 2008 University of Florida Conf. Partitions, q-Series, and Modular Forms.

[2] K. Bringmann and O. Richter, Zagier-type dualities and lifting maps for harmonic Maass-Jacobi forms, Adv. Math 225(4) (2010) 2298-2315.

[3] J. Bruinier and J. Funke, On two geometric theta lifts, Duke Math. J. 125(1) (2004) 45-90.

[4] J. Cardy, Operator content of two-dimensional conformally invariant theories, Nuclear Phys. B 270(2) (1986) 186-204.

[5] M. Eichler and D. Zagier, The Theory of Jacobi Forms (Birkhäuser, Boston, 1985).

[6] B. Gross, W. Kohnen and D. Zagier, Heegner points and derivatives of $L$-series. II, Math. Ann. 278(1-4) (1987) 497-562.

[7] N.-P. Skoruppa, Binary quadratic forms and the Fourier coefficients of elliptic and Jacobi modular forms, J. Reine Angew. Math. 411 (1990) 66-95.

[8] - Developments in the theory of Jacobi forms, in Automorphic Functions and Their Applications (Khabarovsk, 1988) (Acad. Sci. USSR, Inst. Appl. Math., Khabarovsk, 1990), pp. 167-185.

[9] - Explicit formulas for the Fourier coeffcients of Jacobi and elliptic modular forms, Invent. Math. 102(3) (1990) 501-520.

[10] D. Zagier, Note on the Landweber-Stong elliptic genus, in Elliptic Curves and Modular Forms in Algebraic Topology, Lecture Notes in Mathematics, Vol. 1326 (Springer, 1988), pp. 216-224.

[11] S. Zwegers, Mock theta functions, Ph.D. thesis, Universiteit Utrecht, The Netherlands, 2002. 